\title{
Detection of optical linear polarization in the SN 2006aj/XRF 060218 non-spherical expansion ${ }^{\star}$
}

\author{
J. Gorosabel ${ }^{1}$, V. Larionov ${ }^{2}$, A. J. Castro-Tirado ${ }^{1}$, S. Guziy ${ }^{1,3}$, L. Larionova ${ }^{2}$, A. Del Olmo ${ }^{1}$, M. A. Martínez ${ }^{1}$, \\ J. Cepa ${ }^{4}$, B. Cedrés ${ }^{4}$, A. de Ugarte Postigo ${ }^{1}$, M. Jelínek ${ }^{1}$, O. Bogdanov ${ }^{3}$, and A. LLorente ${ }^{5}$ \\ ${ }^{1}$ Instituto de Astrofísica de Andalucía (IAA-CSIC), Apartado de Correos, 3.004, 18080 Granada, Spain \\ e-mail: jgu@iaa.es \\ 2 Astronomical Institute of St. Petersburg University, Petrodvorets, Universitetski pr. 28, 198504, Russia \\ 3 Nikolaev State University, Nikolskaja 24, Nikolaev 54030, Ukraine \\ ${ }^{4}$ Instituto de Astrofísica de Canarias, La Laguna, Tenerife, 38200 Canary Islands, Spain \\ 5 XMM-Newton Science Operations Centre, European Space Agency, Villafranca del Castillo, PO Box 50727, 28080 Madrid, Spain \\ Received 13 September 2006 / Accepted 29 September 2006
}

\section{ABSTRACT}

\begin{abstract}
Aims. We have performed optical polarimetric observations of the SN 2006aj associated to the X-ray flash (XRF) of February 18, 2006, XRF 060218 that provide information on its expansion geometry.

Methods. The data were acquired in the $R$-band with the $0.7 \mathrm{~m}$ telescope of Crimea, $2.5 \mathrm{~m}$ Nordic Optical Telescope and the $2.2 \mathrm{~m}$ of Calar Alto.

Results. We report the detection of linear polarization between 3 and 39 days after the gamma-ray event $\left(t-t_{0}\right)$. This represents the first polarization detection of a Ic supernova (SN) associated to an XRF. Our data exhibit a degree of linear polarization $(P)$ around $P \sim 4 \%$ at $t-t_{0} \sim 3-5$ days, followed by a constant polarization phase with $P \sim 1.4 \%$ at $13.7 \lessgtr t-t_{0} \lesssim 39$ days. Our data suggest a decay in $P$, and more interestingly, show a position angle $(\theta)$ rotation of $\sim 100^{\circ}$ comparing data taken before and after the $R$-band lightcurve peak.

Conclusions. The reported polarization measurements can be explained by the evolution of an asymmetric SN expansion. We discuss on several ingredients that could account for the observed $\theta$ rotation.
\end{abstract}

Key words. gamma rays: bursts - supernovae: general - techniques: polarimetric

\section{Introduction}

The Swift satellite detected a long-soft gamma-ray burst (GRB) at $t_{0}=$ Feb. 18.1492006 UT, (Cusumano et al. 2006), showing both a low redshift $(z=0.033$, Mirabal \& Halpern 2006) and a peak energy (Campana et al. 2006), typical of X-ray flashes (XRF 060218 hereafter). Further observations associated it to a Ic-type supernova, SN 2006aj (Masetti et al. 2006; Soderberg et al. 2006; Pian et al. 2006). This finding strengthened even more the already solid long-duration GRB/Supernova link (Hjorth et al. 2003; Stanek et al. 2003).

Since 1999 (Covino et al. 1999) several GRB afterglows have shown optical polarization at a level of $\sim 1-3 \%$ (see Gorosabel et al. 2004 and references therein). Optical supernovae (SNe), including typical Ic-types not related to GRBs, do not tend to show optical polarization above $\sim 1 \%$. However, some counterexamples exist (Leonard et al. 2006). The very few polarimetric studies of Ic SNe related to GRBs do not allow yet to infer clear global polarimetric differences with normal Ic SNe. However, as we report in this Letter, some normal Ic SNe like

* Based on observations made with the Nordic Optical Telescope, operated on the island of La Palma, in the Spanish Observatorio del Roque de los Muchachos of the Instituto de Astrofísica de Canarias, and on observations done at the Centro Astronómico Hispano Alemán (CAHA) at Calar alto, operated jointly by the Max-Planck Institut für Astronomie and the Instituto de Astrofísica de Andalucía (CSIC).
SN 2002ap (Mazzali et al. 2002) share some similarities with SN 2006aj; polarization levels above $\sim 1 \%$ and, more interestingly, long-term rotations in the polarization angle (Kawabata et al. 2002).

Most of the SN intrinsic polarization detections are attributed to the break of the symmetry around line of sight, due to a Thompson photon scattering through an aspherical SN expansion (Kasen et al. 2003). This polarization is expected to decay as $\sim t^{-2}$, as the atmosphere expands and the optical depth (and hence the polarization) falls (Leonard et al. 2006). In most cases the afterglow is so strong that overshines the SN polarization component (for instance in GRB 030329; Greiner et al. 2003). In this Letter we report the first polarization detection to date of a SN associated to an XRF, not coming from the GRB ejecta forward shock emission (Fan et al. 2006; Soderberg et al. 2006). Section 2 reports the observations carried out for SN 2006aj. Section 3 presents our results and Sect. 4 discusses the implications of our measurements. Section 5 summarises the final conclusions.

\section{Observations and data analysis}

Table 1 displays the log of our observations. All the measurements reported in this paper were carried out in the $R$-band. The observations were performed, chronologically ordered, with 
Table 1. Log of the observations. For the NOT and CAHA the observation are cycles of 4 images, $\left(4 \times T_{\exp }\right)$ with the plate rotator at $0,22.5,45$, 67.5 degrees. The $R$-band magnitudes are calibrated using the standard stars given by Hicken et al. (2006).

\begin{tabular}{ccccccc}
\hline \hline $\begin{array}{c}\text { Days from GRB } \\
t-t_{0}\end{array}$ & $\begin{array}{c}\text { Telescope } \\
\text { (+Instrument) }\end{array}$ & $\begin{array}{c}\text { Exposure Time } \\
\mathrm{s}\end{array}$ & $\begin{array}{c}\text { Seeing } \\
\prime\end{array}$ & $\begin{array}{c}\text { Magnitude } \\
R \text {-band }\end{array}$ & $\begin{array}{c}P \\
\%\end{array}$ & $\begin{array}{c}\theta \\
\text { degrees }\end{array}$ \\
\hline $2.569954-2.597558$ & 0.7 m AZT-8(+ST-7) & $2 \times(10 \times 90)$ & 3.0 & $17.99 \pm 0.03$ & $2.61 \pm 1.80$ & $157.82 \pm 16.47$ \\
$3.579178-3.600764$ & 0.7 m AZT-8(+ST-7) & $2 \times(10 \times 90)$ & 3.8 & $17.88 \pm 0.02$ & $4.53 \pm 1.52$ & $143.64 \pm 7.18$ \\
$4.555046-4.599144$ & 0.7 m AZT-8(+ST-7) & $2 \times(20 \times 90)$ & 3.3 & $17.68 \pm 0.02$ & $4.06 \pm 1.63$ & $143.86 \pm 7.98$ \\
$5.565510-5.612326$ & 0.7 m AZT-8(+ST-7) & $2 \times(20 \times 90)$ & 4.2 & $17.52 \pm 0.02$ & $2.43 \pm 0.89$ & $150.09 \pm 10.02$ \\
$2.569954-5.612326$ & 0.7 m AZT-8(+ST-7) & $2 \times(60 \times 90)$ & 3.5 & $17.71 \pm 0.02$ & $3.40 \pm 1.09$ & $147.56 \pm 7.06^{\star}$ \\
\hline $13.721516-13.755185$ & 2.5 m NOT(+ALFOSC) & $4 \times 700$ & 1.0 & $17.19 \pm 0.04$ & $1.44 \pm 0.14$ & $49.58 \pm 2.73$ \\
$18.673955-18.719135$ & 2.2 m CAHA(+CAFOS) & $4 \times 900$ & 1.6 & $17.38 \pm 0.03$ & $1.40 \pm 0.21$ & $51.62 \pm 4.11$ \\
$38.679248-38.707514$ & 2.2 m CAHA(+CAFOS) & $4 \times 400$ & 2.0 & $18.44 \pm 0.03$ & $<3.9^{\dagger}$ & - \\
\hline
\end{tabular}

$\star$ Determined combining the images of the above four observing epochs. ${ }^{\dagger} 3 \sigma$ upper limit.

the $0.7 \mathrm{~m}$ telescope of Crimea (AZT-8), the $2.5 \mathrm{~m}$ Nordic Optical Telescope (NOT) and the $2.2 \mathrm{~m}$ of Calar Alto (CAHA).

The AZT-8 observations were done with two Savart plates; swapping them the observer can obtain either the Stokes $Q$ (two images are split diagonally) or the $U$ parameter (split horizontally). The field of view (FoV) covered by the AZT- 8 $(+\mathrm{ST}-7 \mathrm{CCD})$ is $8.1^{\prime} \times 5.4^{\prime}$ and the pixel scale is $1.3^{\prime \prime} / \mathrm{pix}$. Since the GRB field is substantially crowded in the AZT- 8 images, we performed PSF photometry (Stetson 1987).

The NOT observations were based on ALFOSC equipped with the FAPOL unit. In order to obtain $Q$ and $U$ with ALFOSC, the GRB field was imaged through a calcite and a $1 / 2$ wave plate. Four images of the GRB field were acquired, rotating the $1 / 2$ wave plate at $0^{\circ}, 22.5^{\circ}, 45.0^{\circ}$ and $67.5^{\circ}$. The calcite plates of FAPOL produce a vignetted field of about $140^{\prime \prime}$ in diameter with a pixel scale of $0.19^{\prime \prime} /$ pix. The CAHA observations were carried with CAFOS. The CAFOS polarization unit is similar to FAPOL (is based on a Wollaston prism instead of a calcite plate), but uses a strip mask on the focal plane to avoid accidental overlapping on the CCD. The total FoV in CAFOS is composed by 14 strips of $9^{\prime} \times 18^{\prime \prime}$ each, containing stars enough for a satisfactory interstellar medium (ISM) polarization correction. For the NOT and CAHA data the determination of $P$ and $\theta$ was carried out fitting the $S(\theta)$ function with the corresponding ISM normalization factor (di Serego Alighieri 1997; see Fig. 1). For both data sets aperture photometry was performed, ranging the aperture radii from 1 pixel to 2.5 times the FWHM (Full Width at Half Maximum). After checking that the magnitudes were independent of the apertures used, the ones yielding minimum errors were adopted.

The Galactic ISM polarization correction was done using 9 (13) field stars on the AZT-8 (CAHA) images. Due to the reduced ALFOSC FoV only one unsaturated bright field star was available in the NOT data. Thus for the NOT images zero (G191B2B) and high (HD 25443) polarization standards allowed us to infer the SN 2006aj Stokes parameters, and for completeness, also the ones of the unique unsaturated field star (which agreed with the ISM law by Serkowski et al. 1975).

\section{Results}

Table 1 shows the position angle $(\theta)$ and the degree of linear polarization $(P)$ of the afterglow once they were corrected by the ISM polarization and also by the fact that $P$ is a positive quantity (multiplying $P$ by $\sqrt{1-\left(\sigma_{P} / P\right)^{2}}$; see di Serego Alighieri 1997). The AZT- 8 results displayed in Table 1 supersede the ones reported previously (Larionov \& Larionova 2006). The four AZT- 8 data sets yield $P$ marginal detections, reaching a

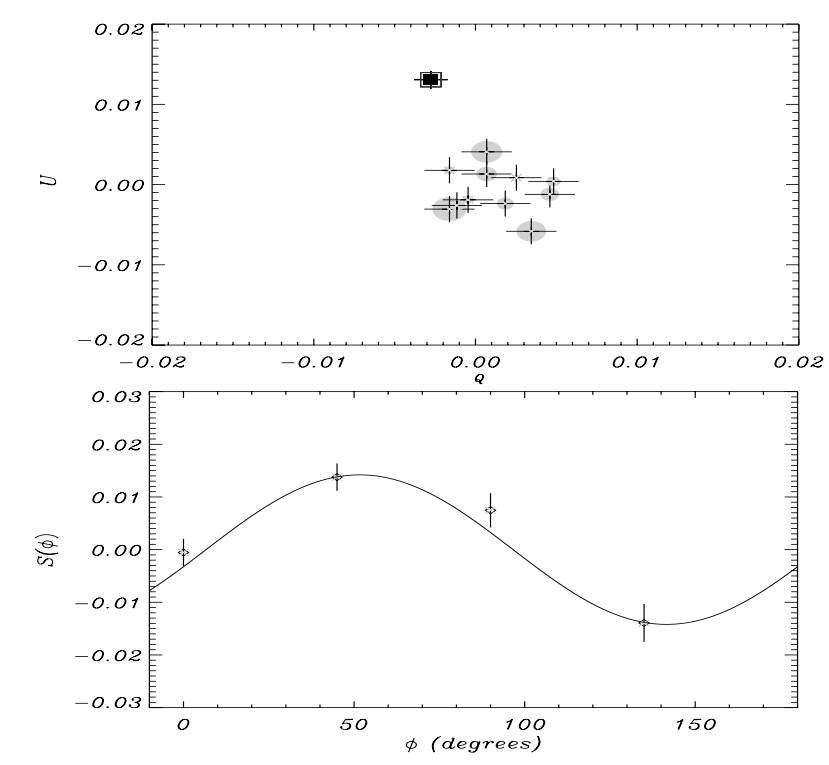

Fig. 1. Upper panel: the $Q, U$ plane of the stars in the field (circles) of XRF 060218 (square) imaged from CAHA on March 8.845 UT $\left(t-t_{0}=18.7\right.$ days). The size of each circle is proportional to the distance of the corresponding star to the center of the CCD. This is used to check that the instrumental polarization, if present, does not vary severely across the FoV. Lower panel: the satisfactory fit of the $S(\phi)$ function $\left(\chi^{2} /\right.$ d.o.f. $\left.=1.38\right)$. As shown the XRF 060218/SN 2006aj is clearly polarized. An aperture radius $=1 \mathrm{FWHM}$ was assumed.

$3 \sigma$ confidence level at $t-t_{0} \sim 3.6$ days. The apparently large $P$ variations (from $P=2.43$ to 4.53 ) in the first four nights are within $P$ errors, so we they are likely statistical fluctuations. However, we note that for the four epochs SN 2006aj is systematically placed at the same Stokes plane area, once the ISM correction is included. In order to reinforce the $P$ detection, the four AZT-8 observing epochs were co-added and PSF photometry performed. The fifth line of Table 1 , shows the result when the four AZT- 8 epochs are combined. The estimate of the $P$ and $\theta$ errors in the AZT- 8 images were obtained modelling with a Montecarlo method the flux error distribution of SN 2006aj and the 9 field stars used for the ISM correction. Figure 2 shows the Stokes plane when all the AZT- 8 data are combined. The cloud of dots representing the SN 2006aj $(Q, U)$ distribution is clearly off the center defined by the field stars. The $P$ value inferred from the combined image is $3.40 \pm 1.09 \%$, detected at a $3.1 \sigma$ level (including the $\sqrt{1-\left(\sigma_{P} / P\right)^{2}}$ term $)$.

Both the NOT and first CAHA data show conclusive $P$ detections with confidence levels of $10.3 \sigma$ and $6.7 \sigma$ respectively. The 


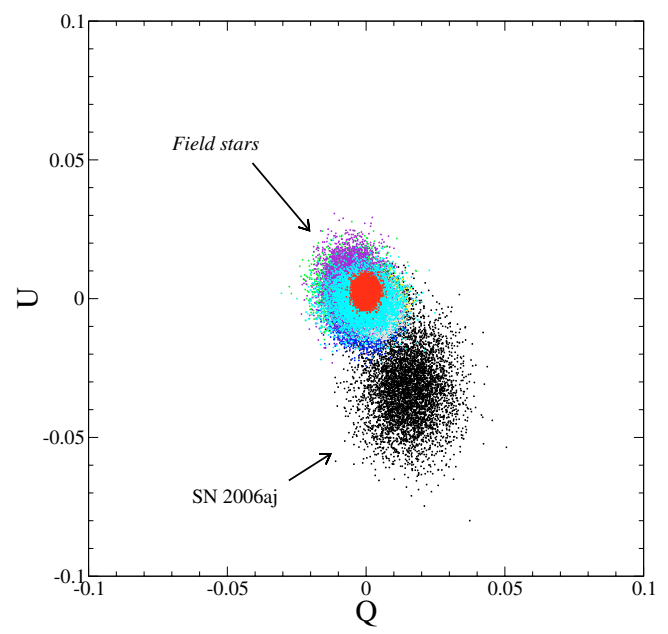

Fig. 2. The Stokes plane of 9 field stars and SN 2006aj on the combined AZT-8 image when the Galactic ISM correction is applied. The clouds of dots were created altering with a Montecarlo method the fluxes of the objects according to their photometric errors. The cloud representing SN 2006aj is shifted with respect to the origin defined by the field stars, assumed to be intrinsically unpolarized in average.

$\theta$ values of both epochs agree with a stable angle around $50^{\circ}$, but interestingly it appears rotated by $\sim 100^{\circ}$ with respect to the AZT-8 images. Our data points reject a stationary $P$ value at a $3 \sigma$ level, so we conclude that $P$ likely fades at $5.6<t-t_{0}<$ 13.7 days. Such a $P$ decay resembles the late evolution of the asymmetric SN 2004dj (Leonard et al. 2006), which showed an abrupt $P$ peak at $t-t_{0} \sim 90$ days due to the sharp appearance of the inner ejecta, followed by a late $\sim t^{-2}$ decay.

We point out that the result of our $R$-band polarimetry is a weighted mean of the continuum and the spectral features (in principle each one with a different polarization) falling inside the wavelength range of the filter transmittance (centred at $6500 \AA$ ). With no spectro-polarimetric data it is not possible a priory to quantify if the measured polarization evolution is dominated by spectrally localized large polarization fluctuations. We note however that the SN 2006aj spectra around $6500 \AA$ are smooth. Thus the $\theta$ rotation measured at $5.5<t-t_{0}<13.7$ days, is not easily explainable by colour evolution, which occurs at wavelengths bluer than $5000 \AA$ in that epoch range (Mazzali et al. 2006).

\section{Discussion}

The most natural framework to explain our polarization measurements is a non spherical SN expansion, consistent with the scenario proposed for XRF 060218 by recent studies (Pian et al. 2006). It is interesting to note that other Ic SNe exhibiting nonspherical expansions have been linked to long-duration GRBs (Höflich et al. 1999). Furthermore there is growing evidence that an important fraction of Ic SNe are triggered by bipolar ejections (Granot \& Ramirez-Ruiz 2004). It is widely accepted that non-zero SN polarization measurements demand some degree of expansion asphericity. If the projection of the SN along the line of sight is elliptical, a non canceled linear polarization should be present parallel to the minor axis (Kasen et al. 2003). An ellipsoidal expansion is fairly consistent with the measured $P$ (see $t^{-2}$ curve of Fig. 3). However, a single ellipsoidal model can not account for the measured $\theta$ rotation.

The decay in $P$ seems to be accompanied with a decay of the photospheric expansion velocity (notice the similarity between our Fig. 3 lower panel and Fig. 2 of Pian et al. 2006).

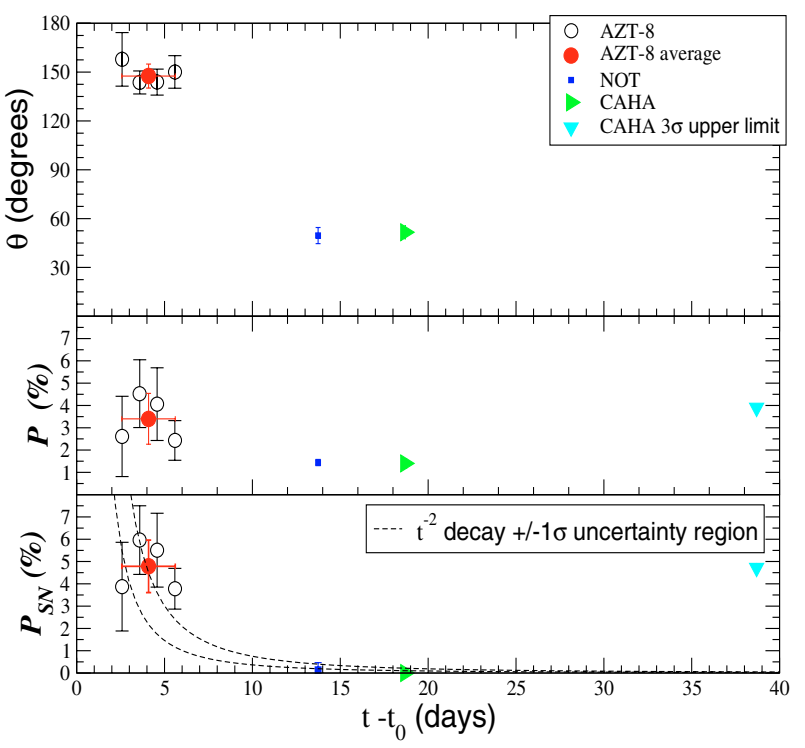

Fig. 3. Our polarization results, once the the Galactic ISM correction was considered. Upper panel: as shown $\theta$ exhibited a rotation of $\sim 100^{\circ}$ at $5.6<t-t_{0}<13.7$ days. Middle panel: $P$ shows a likely decay reaching a constant level at a $\sim 1.4 \%$ level. Lower panel: the intrinsic $P_{\mathrm{SN}}$ evolution assuming that our last $P$ detection is entirely originated by the host ISM. The dashed curves show the $\pm 1 \sigma$ uncertainty region of the $t^{-2}$ decay, as seen in SN 2004dj.

This possible correlation between the photospheric expansion speed and the polarization, might imply the existence of a fast $\left(v>20000 \mathrm{~km} \mathrm{~s}^{-1}\right.$, Pian et al. 2006) highly asymmetric ejecta responsible of the high polarization measured 2.6-5.6 days after the GRB. This rapid asymmetric ejecta might be aligned along the GRB jet. Several days later a slower $\left(v<15000 \mathrm{~km} \mathrm{~s}^{-1}\right.$, Pian et al. 2006) SN bulk ejecta would circularice the geometry, which would explain the lower $P$ of our CAHA and NOT measurements. We could speculate that this slower ejecta might be in a toroidal-shape equatorial expansion, so the geometric transition from the rapid to slow ejecta might naturally explain the detected rotation of $\sim 100^{\circ}$. These results partially resemble the jetlike geometry proposed for SN 2002ap, which exhibited lower $P$ values than SN 2006aj, but with a $\theta$ rotation similar to it $\left(\sim 70^{\circ}\right.$; Kawabata et al. 2002). However, other ingredients can also induce a rotation in $\theta: i$ ) the host galaxy ISM; $i i)$ a non radial distribution of ${ }^{56} \mathrm{Ni}$; and iii) a dusty circumstellar medium (CSM). These effects can be combined producing a complex picture.

As discussed by Klose et al. (2004) the polarization measured in afterglows are sensitive to the host galaxy ISM, so it might well affect our observations. Thus, the $\theta$ stability at $t-t_{0}>$ 13.7 days could be the result of the $P$ induced by the host ISM, so the SN 2006aj intrinsic polarization $\left(P_{\mathrm{SN}}\right)$ would dominate just before our NOT data point. According to the Serkowski's law $E(B-V) \geq 0.15$ would be required in the host line of sight to account for the $P \sim 1.4 \%$ NOT and CAHA measurements. This $E(B-V)$ value agrees fairly well with the host extinction estimated by Modjaz et al. (2006; $E(B-V) \sim 0.05-0.11)$, Sollerman et al. (2006; $E(B-V) \sim 0.1-0.3)$, and Campana et al. (2006; $E(B-V)=0.2)$. We note however the lower estimate by Guenther et al. $\left(2006 ; E(B-V)=0.042^{1}\right)$, so the extinction although present, might not account totally for the late polarization. In this Framework the $\theta$ change of $\sim 100^{\circ}$ would just obey

1 See Sollerman et al. (2006) for a discussion on possible uncertainties and biases affecting this measurement. 
to the chance alignment between the asymmetric expansion and the linear polarization induced by the host ISM. Assuming that our last $P$ detection $\left(t-t_{0} \sim 18.7\right.$ days) is purely due the host ISM, the SN 2006aj intrinsic polarization at $t-t_{0} \sim 4.5$ days would be higher $\left(P_{\mathrm{SN}}=4.78 \pm 1.17 \%\right.$, see Fig. 3 lower panel $)$.

An asymmetric distribution of ${ }^{56} \mathrm{Ni}$ could alter the $\mathrm{SN}$ radiation field, specially after the lightcurve peak when the ${ }^{56} \mathrm{Ni}$ radioactive decay powers the optical lightcurve. The collapsar model predicts a subrelativistic disk wind of neutron and protons, which after cooling would form ${ }^{56} \mathrm{Ni}$ (Woosley \& Bloom 2006). A picture to explain the $\theta$ rotation of $\sim 100^{\circ}$, would require an equatorial deposition of synthesized ${ }^{56} \mathrm{Ni}$, which would alter the $\theta$ measured after the lightcurve peak.

Another possible mechanism to explain the observed $\theta$ change could be delayed CSM scattered light. This process was proposed to interpret the SN 1987A polarization lightcurve (Wang \& Wheeler 1996). In this scenario the polarization would consist of two components, the one due to photons propagating directly from the (possibly aspherical) $\mathrm{SN}$, and the second one from scattered light by a dusty CSM region. The epochs of our observations would push the CSM scattering region further than $\sim 2.3$ light-days from the progenitor. However, this process should have originated a clear distortion of the spectra at $\lambda \gtrsim 6000 \AA$ after the lightcurve peak, which is not observed (Modjaz et al. 2006; Pian et al. 2006).

\section{Conclusions}

We report the detection of $R$-band linear polarization for XRF 060218/SN 2006aj in observations done since $t-t_{0} \sim 3$ to 39 days. These observations represent the first detection of optical polarization in an XRF. The measured high degree of polarization gives further credence to the XRF/GRB off-axis scenario, since a face-on SN would not likely produce any intrinsic polarization. As Patat et al. (2001) noted there is a degeneracy between the viewing angle and the asymmetry level, so a detailed geometric picture based on only our data is not possible. Our data show a $P \sim 4 \%$ value in the first $\sim 3-5$ days after the GRB, followed by a constant $P \sim 1.4 \%$ polarization phase at $t-t_{0} \gtrsim 13.7$ days, suggesting a $P$ decay. $\theta$ shows a $\sim 100^{\circ}$ rotation at $5.6<t-t_{0}<13.7$ days. We propose that the $P$ evolution can be explained by an asymmetric SN. We discuss several ingredients which separately, or combined, can explain the observed $\theta$ rotation (in decreasing order of relevance); i) a highly asymmetric high-velocity SN ejection, followed by a less asymmetric SN bulk; ii) polarization induced by the host ISM; iii) asymmetric distribution of ${ }^{56} \mathrm{Ni}$; and iv) scattering by a dusty region placed a few light-days from the progenitor.

Acknowledgements. This study is supported by Spanish research programmes ESP2002-04124-C03-01 and AYA2004-01515. JG thanks the hospitality of the Donostia International Physic Center (DIPC). We thank our anonymous referee for helpful comments.

\section{References}

Campana, S., Mangano, V., Blusin, A. J., et al. 2006, Nature, 442, 1008 Covino, S., Lazzati, D., Ghisellini, G., et al. 1999, A\&A, 348, L1 Cusumano, G., Barthelmy, S., Gehrels, N., et al. 2006, GCN, 4775 di Serego Alighieri, S. 1997, in Instrumentation for Large Telescopes, ed. J. M. Rodríguez Espinosa, A. Herrero, \& F. Sanchéz (Cambridge Univ. Press), 287

Fan, Y. Z., Piran, T., \& Xu, D. 2006, J. Cosmol. Astropart. Phys., 9, 13 Gorosabel, J., Rol, E., Covino, S., et al. 2004, A\&A, 422, 113 Granot, J., \& Ramirez-Ruiz, E. 2004, ApJ, 609, L9

Greiner, J., Klose, S., Reinsch, K., et al. 2003, Nature, 426, 157 Guenther, E. W., Klose, S., Vreeswijk, P. M., et al. 2006, GCN, 4863 Hicken, M., Modjaz, M., Challis, P., et al. 2006, GCN 4898 Hjorth, J., Sollerman, J., Moller, P., et al. 2003, Nature, 423, 847 Höflich, P., Wheeler, J. C., \& Wang, L. 1999, ApJ, 521, 179

Kasen, D., Nugent, P., Wang, L., et al. 2003, ApJ, 593, 788 Kawabata, K. S., Jeffery, M., Ohyama, Y., et al. 2002, ApJ, 580, L39 Klose, S., Palazzi, E., Masetti, N., et al. 2004, A\&A, 2004, 420, 899 Larionov, V., \& Larionova, L. 2006, GCN 4836 Leonard, D. C., Filippenko, A. V., Ganeshalingam, M., et al. 2006, Nature, 440, 505

Masetti, N., Palazzi, E., Pian, E., et al. 2006, GCN, 4803

Mazzali, P. A., Deng, J., Maeda, K., et al. 2002, ApJ, 572, L61

Mazzali, P. A., Deng, J., Nomoto, K., et al. 2006, Nature, 442, 1018 Mirabal, N., \& Halpern, J. 2006, GCN 4792

Modjaz, M., Stanek, K. Z., Garnavich, P., et al. 2006, ApJ, 645, L21

Patat, F., Cappellaro, E., Danziger, J., et al. 2001, ApJ, 555, 900

Pian, E., Mazzali, P. A., Masetti, N., et al. 2006, Nature, 442, 1011

Serkowski, K., Mathewson, D. S., \& Ford, V. L. 1975, ApJ, 196, 261

Soderberg, A., Kulkarni, S. R., Nakar, E., et al. 2006, Nature, 442, 1014

Sollerman, J., Jaunsen, A. O., Fynbo, J., et al. 2006, A\&A, 454, 503

Stanek, K. Z., Matheson, T., Garnavich, P., et al. 2003, ApJ, 591, L17

Stetson 1987, PASP, 99, 191

Wang, L., \& Wheeler, J. C. 1996, ApJ, 462, L27

Woosley, S. E., \& Bloom, J. S. 2006, ARA\&A, 44, 507 